\title{
Addendum: On sets of maximum indetermination of analytic functions
}

By

\section{E. F. COLLINGWOOD}

In my paper published in Math. Zeitschr. vol. 67, pp. 377-396 (1957) there is an error, kindly pointed out to me by Dr. J.D. WESTON, in the proof of Theorem 7, where the assertion that the inequality (10) holds uniformly in the set $H\left(\vartheta,-\eta_{L}\right)$ is invalid. To obtain a valid proof I replace the passage which begins with the sentence containing (10) by the following argument:

$E_{N, M, L}$ is therefore dense in an arc of the circumference $\varkappa$ which we may clearly suppose to be an open arc $\left(\vartheta_{1}, \vartheta_{2}\right)$ of angular measure less than $\eta_{L}$. We can find $e^{i \vartheta} \in E_{N, M, L} \cap\left(\frac{\vartheta_{1}+\vartheta_{2}}{2}, \vartheta_{2}\right)$ so that $\left(\vartheta_{1}, \frac{\vartheta_{1}+\vartheta_{2}}{\dot{2}}\right)<\left(\vartheta_{1}, \vartheta\right)<\left(\vartheta-\eta_{L}, \vartheta\right)$; and since $H(\vartheta)$ is dense in $\left(\vartheta-\eta_{L}, \vartheta\right)$ we can find $e^{i \vartheta^{\prime}} \in H(\vartheta) \cap\left(\vartheta_{1}, \frac{\vartheta_{1}+\vartheta_{2}}{2}\right)$. By the definition of $E_{N, M, L}$ we have $a \in \mathscr{C}\left(\bar{C}\left(f, H\left(\vartheta,-\eta_{L}\right)\right)_{+\varepsilon_{N} / 2}\right)$ and hence $a \in \mathscr{C}\left(C\left(f, e^{i \vartheta^{\prime}}\right)_{+\varepsilon_{N} / 2}\right)$. The point $e^{i \vartheta^{\prime}}$ is therefore the centre of a circle $\delta\left(\vartheta^{\prime}\right)$ such that the inequality

$$
[f(z), a]>3 \varepsilon_{N} / 8
$$

is satisfied for all $z \in \delta\left(\mathscr{\vartheta}^{\prime}\right),|z|<1$. For if not we can find a sequence $\left\{z_{n}\right\}$, $\left|z_{n}\right|<1, \lim _{n \rightarrow \infty} z_{n}=e^{i y^{\prime \prime}}$ such that $\left[f\left(z_{n}\right), a\right] \leqq \varepsilon_{N} / 3$ which implies that

$$
a \in C\left(f, e^{i \vartheta^{\prime}}\right)_{+3 \varepsilon_{N} / 8}<C\left(f, e^{i \vartheta^{\prime}}\right)_{+\varepsilon_{N} / 2} \text {. }
$$

If now $\gamma$ is an arc of $x$ interior to $\delta\left(\vartheta^{\prime}\right)$ it follows from (10) that for all $e^{i \vartheta^{\prime \prime}} \in \gamma$ we must have

$$
a \in \mathscr{C}\left(C\left(t, e^{i \vartheta^{\prime \prime}}\right)_{+\varepsilon_{N} / 3}\right)
$$

and hence $T_{M} \cap C\left(f, e^{i \theta^{\prime \prime}}\right)$ is empty since $T_{M}$ is of diameter less than $\varepsilon_{N} / 4$. But since $E_{N, M, L}$ is dense in $\gamma$ and is a subset of $E_{N, M}$ we can choose $e^{i \vartheta^{\prime \prime}} \in E_{N, M} \cap \gamma$. But with this choice of $\vartheta^{\prime \prime}$, by the definition of $E_{N, M}$ above,

$$
T_{M} \cap C\left(f, e^{i \vartheta^{\prime \prime}}\right) \cap \mathscr{C}\left(C_{H r}\left(f, e^{i \vartheta^{\prime \prime}}\right)_{+\varepsilon_{N}}\right)
$$

is not empty and therefore the larger set $T_{M} \cap C\left(f, e^{i \vartheta^{\prime \prime}}\right)$ is not empty. We have thus reached a contradiction which proves the theorem.

It will be noted that the reconstructed proof is independent even of continuity. It follows that Theorem 7 and Corollary 3 are valid for an arbitrary 
function defined in the interior of the unit disc $|z|<1$. This places them on the same footing as a recent theorem of BAGEMIHL [Proc. Nat. Acad. Sci. USA 41, 379-382 (1955), Theorem 2] on the intersection of pairs of internal curviliear cluster sets of an arbitrary function $f(z)$. An argument of Gross [Math. Zeitschr. 2, 249-250 (1918)] at once shows BAGEminL's result to be applicable also to the intersection of the right and left-hand boundary cluster sets $C_{B r}\left(f, e^{i \vartheta}\right) \cap C_{B l}\left(f, e^{i \vartheta}\right)$ which is therefore not empty except perhaps at an enumerable set of points $e^{i \theta} \in \%$, while the generalised Corollary 3 shows that this intersection is actually maximal except perhaps in a set of the first category on $x$.

Lilburn Tower, Alnwick, Northumberland (England)

(Eingegangen am 4. November 1957) 